\title{
Management of Pancreatic Calculi - an Experience of Ten Cases in a District Hospital
}

\author{
Md. Rafiqul Islam*1, Kazi Moinur Rahman², Gazi Shafiqur Rahman³
}

\section{Abstract}

Introduction: Pancreatic calculi $(P C)$ are not an uncommon surgical condition in daily practice.Alcohol, smoking, genetic factors, metabolic disturbances and defects in immunity are some of the known etiological agents. The common presentations are severe abdominal pain, vomiting, malabsorbtion, weight loss, diabetes mellitus etc. Case Report: We have managed ten cases in our hospital,Initial medical treatment was given to all of the patients, followed by open surgical procedure. Discussion: Endoscopic stone removal is the best procedure in a higher center. Open surgical procedure is needed in some cases. Conclusion: We have done ten cases of open pancreatolithotomy in our district hospital. The outcome of the procedure is satisfactory.

Keywords: Pancreatic calculi (PC), Chronic pancreatitis (CP), Pancreaticojejunosetomy.

Number of Figures: 03; Number of References: 10; Number of Correspondence: 03.

*1. Corresponding Author:

Dr. Md. Rafiqul Islam, FCPS(S)

Senior Consultant

Department of Surgery

General Hospital Khulna.

Email: dr.rafiqrmc23@gmail.com

2. Dr. Kazi Moinur Rahman, MS(Ortho Surgery)

Senior Consultant

Department of Ortho. Surgery

General Hospital Khulna.

3. Dr. Gazi Shafiqur Rahman, MBBS

Assistant Registrer, Surgery

General Hospital Khulna.

\section{Introduction:}

Chronic pancreatitis (CP) is a disease of diverse etiology characterized by progressive and irreversible changes in the pancreas, resulting in loss of exocrine and endocrine functions. Pain, either continuous or episodic, is the dominant and distressing feature of this illness and significantly worsens the quality of life. Alcohol, smoking, genetic factors, metabolic disturbances and defects in immunity are some of the known etiological agents ${ }^{1}$. While alcohol is the commonest etiological agent in most industrialized countries, the nonalcoholic idiopathic type of $\mathrm{CP}$ is more prevalent in some countries ${ }^{2-4}$. Pancreatic calculi (PC) are the sequelae of CP and can occur in about $50 \%$ of patients ${ }^{5}$. These calculi aggravate or produce the typical pancreatic pain experienced by patients, by obstructing pancreatic ducts and producing upstream ductal hypertension and subsequent parenchymal hypertension. Therapy, either endoscopic or surgical aims at clearing these calculi and reducing the ductal hypertension, relieving pain and improving quality of life. PC seen in the nonalcoholic, idiopathic variety of $\mathrm{CP}$ tend to be large and denser than those seen in the alcoholic variety ${ }^{6,7}$.

\section{Case Report: Classification of Pancreatic Calculi}

PCs are classified on the basis of type, numbers and location. They may be (1) radio opaque, radiolucent or mixed; (2) single or multiple; (3) located in the main pancreatic duct (MPD), side branches or in the pancreatic parenchyma; and (4) located in head, body or tail regions (Fig. 1). Majority of PCs are radio opaque while a few are radiolucent or mixed ${ }^{2}$.

\section{Diagnosis}

Patient with pancreatic calculi usually presents with characteristic abdominal pain, associatedwith vomiting. A plain $\mathrm{x}$-ray of abdomen is sometime enough to diagnose the case. There are radiopaque shadows present at the level of body of L1-L2 or on either side. Other investigations are Complete blood $\mathbf{F} / \mathbf{P}$ and Lateral view. Showing radio-opaque count, Blood sugar level, Serum shadows of pancreatic stone.

creatinine, Blood grouping, Serum

amylase / lipase, Ultrasonography of abdomen, CT Scan, MRI, ERCP etc.

Further investigations are necessary to diagnose the comorbidities and anaesthetic fitness such as x-ray chest, ECG, Echocardiography etc.

\section{Pathogenesis and Composition}

Pancreatic stone protein (PSP) plays a key role in the formation of $\mathrm{PC}^{8}$. Various factors including gene expression, cause a reduction in PSP. Reduction in PSP results in supersaturation of calcium carbonate in the pancreatic juice. This calcium carbonate is then deposited over an inner nidus. Irrespective of the etiology of $\mathrm{CP}$, the structure and composition of PC are the same suggesting a common pathway for pancreatolithiasis 9 .

Scanning electron microscopy and energy dispersive X-ray fluorescence have revealed that all PC have an amorphous nidus, which forms the center of the PC. The nidus contains elements such as nickel, iron and chromium. It is over this nidus that calcium carbonate in the form of 
calcite is deposited in multiple layers and over multiple stages ${ }^{9}$.

\section{Modalities of Treatment}

As mentioned earlier pain is the dominant symptom in patients with $\mathrm{CP}$ and calculi contribute by obstructing the pancreatic ducts and increasing upstream hypertension. A coexisting ductal stricture can exacerbate the preexisting hypertension.

The following are the modalities of therapy for removal of $\mathrm{PC}$.

1. Endoscopic therapy: this includes endoscopic retrograde cholangiopancreatography (ERCP) and stone extraction and ESWL.

2. Surgical therapy: both drainage and resection procedures are widely used in the surgical management of $\mathrm{CP}$ with calculi.

3. Dissolution of PC: even though chemical agents such as trimethadione have been earlier shown to dissolve stones, these are seldom used in modern day practice.

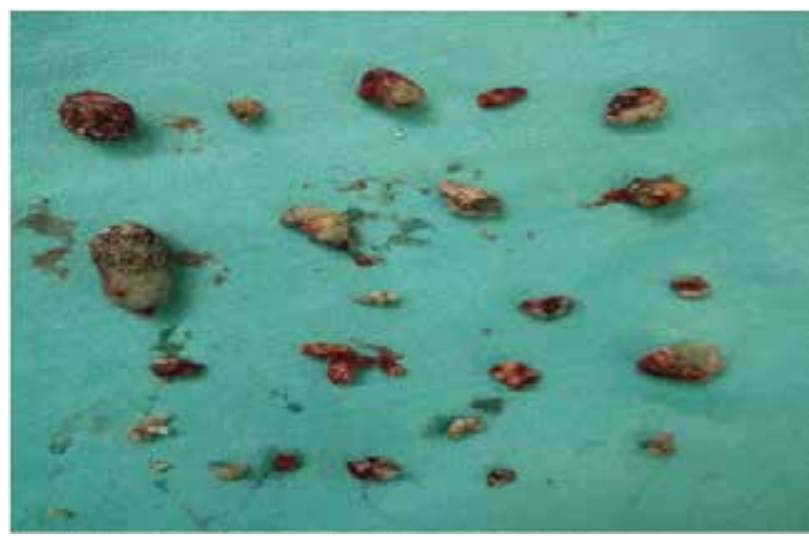

Figure-2: Pancreatic calculi.

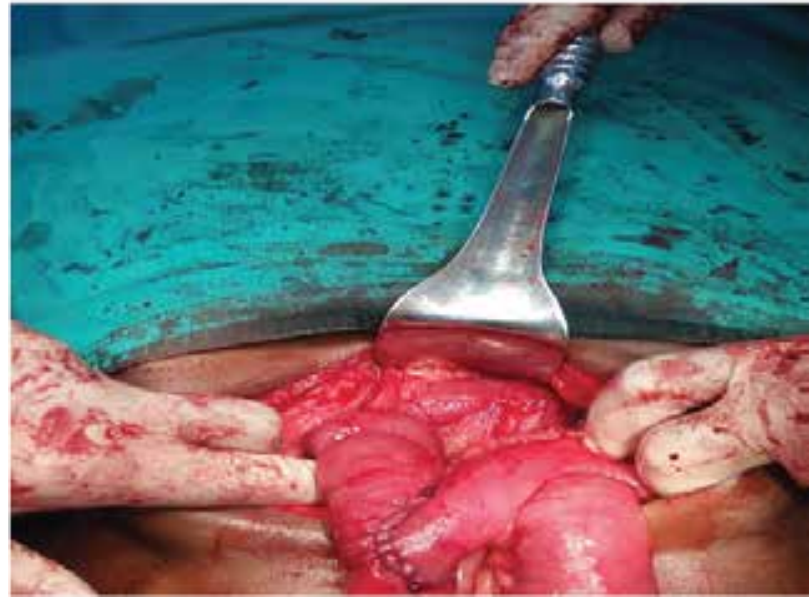

Figure-3: Lateral pancreatojejunosetomy with Roux-en-Y anastomosis.

\section{Discussion:}

We have treated ten cases of pancreatic calculi. All the cases were diagnosed by careful history taking, clinical examination with relevant investigations. Out of ten cases one is female patient. Ages are ranging from 22 years to
65 years. Initially conservative medical treatment was given to every patient but no significant improvement was there. Patient with multiple stones (Fig.2) and dilated main pancreatic duct were selected for open surgical procedure. With all aseptic precaution laparotomy was done, lateralpancreaticojejunosetomy with Roux-en-y anastomosis (Fig.3) were done in six cases, lateral pancreaticojejunosetomy with jejunojejunosetomy were done in two cases, Shahid procedure (lateral side to sidepancreaticojejunosetomy) were done in two cases. The outcome of the treatment were satisfactory, four patients need two or three visits postoperatively for abdominal pain, which were improved on conservative treatment.

\section{Conclusion:}

$\mathrm{PC}$ are the natural sequel of the ongoing process of CP. Pain is the dominant symptom of patients with $\mathrm{CP}$ and various endoscopy and surgical therapy aims at reducing this pain by eliminating the calculi. Small PC are cleared by the standard technique of pancreatic sphincterotomy (PS) followed by balloon trawl or basket. Large calculi in uncomplicated patients should be subjected to ESWL for fragmentation prior to a subsequent ERCP. In properly selected patients ESWL is an efficient and useful tool and provides adequate long-term relief ${ }^{10}$. Patients with extensive calculi, multiple strictures, suspicious mass lesions and those who have failed endotherapy are ideal candidates for surgery. The outcome of our cases after long term follow up is satisfactory.

\section{Consent}

Prior informed consent was obtained from the patient for publication of this case study and any accompanying images. All images are taken by the authors won cell phone during operation.

\section{Conflict of Interest: None.}

\section{Acknowledgement:}

The authors gratefully acknowledge Dr. Ridwanul Islam MBBS for helping with valuable suggestions and documentation.

\section{References:}

1. Lankisch PG, Banks PA. Pancreatitis. New York: Springer-Verlag; 1998.

2. Tandan M, Reddy DN, Santosh D. Extracorporeal shock wave lithotripsy and endotherapy for pancreatic calculi: a large single center experience. Indian $\mathbf{J}$ Gastroenterol. 2010; 29: 143-148. doi: 10.1007/s12664-010-0035-y.

3. Bhasin DK, Singh G, Rana SS. Clinical profile of idiopathic chronic pancreatitis in North India. ClinGastroenterolHepatol. 20097594-599. doi: 10.1016/j.cgh.2009.01.009.

4. Balakrishnan V, Unnikrishnan AG, Thomas V. Chronic pancreatitis: a prospective nationwide study of 1,086 subjects from India. JOP. 2008; 9: 593-600. 
5. Rosch T, Daniel S, Scholz M. Endoscopic treatment of chronic pancreatitis: a multicenter study of 1000 patients with long-term follow-up. Endoscopy. 2002; 34: 765-771. doi: $10.1055 / \mathrm{s}-2002-34256$.

6. Ong WC, Tandan M, Reddy V, Rao GV, Reddy N. Multiple main pancreatic duct stones in tropical pancreatitis: safe clearance with extracorporeal shockwave lithotripsy. Gastroenterol Hepatol. 2006; 21:1514-1518. doi: 10.1111/j.1440-1746.2006.04224.x.

7. Chari S, Jayanthi V, Mohan V, Malathi S, Madanagopalan N, Viswanathan M. Radiological appearance of pancreatic calculi in tropical versus alcoholic chronic pancreatitis. J Gastroenterol Hepatol. 1992; 7: 42-44. doi: 10.1111/j.1440-1746.1992.tb00932.x.

8. Jin CX, Naruse S, Kitagawa M. Pancreatic stone protein of pancreatic calculi in chronic calcified pancreatitis in man. JOP. 2002; 3: 54-61.

9. Pitchumoni CS, Viswanathan KV, Gee Varghese PJ, Banks PA. Ultrastructure and elemental composition of human pancreatic calculi. Pancreas. 1987; 2: 152-158. doi: 10.1097/00006676-198703000-00005

10. Tandan M, Nageshwar Reddy D. Endotherapy in chronic pancreatitis. World J Gastroenterol. 2013; 19: 6156-6164. doi: 10.3748/wjg.v19.i37.6156. 